\title{
LIBERTAD DE EXPRESIÓN Y DERECHO DE DEFENSA FRENTE AL IUS PUNIENDI DE LA ADMINISTRACIÓN: UNA VISIÓN A LA LUZ DE LA MÁS RECIENTE JURISPRUDENCIA CONSTITUCIONAL
}

\author{
FREEDOM OF EXPRESSION AND RIGHT OF DEFENSE OPPOSITE TO THE IUS \\ PUNIENDI OF THE ADMINISTRATION: A VISION IN THE LIGHT OF THE \\ MOST RECENT CONSTITUTIONAL JURISPRUDENCE
}

\author{
José Mateos Martínez*
}

RESUMEN: El presente artículo analiza el reforzamiento de la libertad de expresión que se produce cuando ésta es ejercida en conexión con el derecho de defensa, y se centra en un concreto supuesto que ha sido recientemente examinado por el Tribunal Constitucional: el ejercicio del derecho de defensa en primera persona por un funcionario que es objeto de un expediente disciplinario. A la vez que estudiamos la solución dada por el TC al citado caso, reflexionamos sobre los efectos de la misma más allá del caso específico que resuelve, planteando la posibilidad de su extrapolación a la generalidad de supuestos donde el ciudadano ejerce su derecho de defensa en primera persona y sin asistencia letrada.

\begin{abstract}
The present article analyzes the reinforcement of the freedom of expression that takes place when this one is exercised in connection by the right of defense, and centres on a concrete supposition that has been recently examined by the Constitutional Court: the exercise of the right of defense in the first person for a civil servant who is an object of a disciplinary process. Simultaneously that we study the solution given by the Constitutional Court to the mentioned case, we think about the effects of the same one beyond the specific case that resolves, raising the possibility of its extrapolation to the generality of suppositions where the citizen exercises his right of defense in the first person and without legal aid service.
\end{abstract}

PALABRAS CLAVE: libertad de expresión, derecho de defensa, funcionario público, autotutela, expediente disciplinario.

KEYWORDS: freedom of expression, right of defense, civil servant, autoguardianship, disciplinary process.

Fecha de recepción: 06/02/2017

Fecha de aceptación: 17/04/2017

doi: https://doi.org/10.20318/universitas.2017.3749

\footnotetext{
* Profesor Asociado de Derecho Constitucional en la Universidad de Murcia. Doctor Europeo en Derecho Constitucional por la Universidad de Bolonia y Abogado en ejercicio. E-mail: jmm21@um.es
} 


\section{1.- UNA PERSPECTIVA GENERAL}

Dada la ductilidad de los derechos fundamentales, cuyo único límite se encuentra en el respeto a su contenido esencial ${ }^{1}$, resulta posible que el ámbito de facultades amparado por uno de estos derechos se expanda cuando su ejercicio se interconecta con otro derecho fundamental o principio constitucional que lo justifica².

Así sucede con el derecho fundamental a la libertad de expresión cuando se ejerce como herramienta para hacer valer otros derechos fundamentales, como puede ser el derecho de defensa consagrado en el art. 24.2 CE.

Es decir, en un contexto donde la libertad de expresión es ejercida no solamente para exteriorizar ideas u opiniones, sino también para proteger los derechos e intereses legítimos de un ciudadano en el marco de un procedimiento judicial o administrativo donde éstos pueden verse gravemente dañados en caso de no gozar de una defensa adecuada, la libertad de expresión se expande y goza de un singular ámbito de protección, dada la pluralidad, relevancia y amplitud de derechos en juego.

\section{2.- LIBERTAD DE EXPRESIÓN Y DERECHO DE DEFENSA EN EL EJERCICIO DE LA ASISTENCIA LETRADA}

La principal manifestación de la interconexión entre los derechos fundamentales de libertad de expresión y de defensa, se

1. Sobre el valor del contenido esencial de los derechos fundamentales como esfera de lo indecidible, es decir, como conjunto de bienes y facultades elementales que ninguna decisión del legislador podrá negar, resulta singularmente clara la siguiente exposición de Prieto Sanchís: "Como toda norma constitucional, los derechos ostentan un contenido prescriptivo que resulta inaccesible al legislador; si la Constitución proclama la libertad religiosa o la garantía del imputado de no declarar contra sí mismo, ello significa que ninguna ley puede impedir el ejercicio de aquella libertad -v. gr. haciendo obligatoria la misa dominical- o la efectividad de aquélla garantía -v.gr. autorizando la tortura-. Y esto con total independencia de que eventualmente existan buenas razones políticas, o la mayoría parlamentaria así lo considere, para limitar, suspender o suprimir los derechos correspondientes. Sencillamente, no puede hacerlo porque la fuerza normativa de la Constitución impide que el legislador ordinario o cualquier otro poder público someta a debate lo que ha decidido el poder constituyente" (L. Prieto Sanchís, "La limitación de los derechos fundamentales y la norma de clausura del sistema de libertades", Derechos y libertades: Revista del Instituto Bartolomé de las Casas, Año no 5, no 8, 2000, p. 430).

2. Así sucede con la libertad de expresión cuando se proyecta sobre asuntos de relevancia pública, como puede ser la mala gestión de un representante político. En tal contexto, la libertad del ciudadano que critica el proceder del gestor público se expande y ampara expresiones de singular dureza que no estarían justificadas si se dirigiesen contra un particular. El bien jurídico que constituye el debate libre sobre los asuntos públicos (sin el cual la corrupción generalizada está asegurada por mor del oscurantismo en que la sociedad caería) justifica ese plus de libertad. En tal sentido, vid. C. López Redondo, La libertad de expresión en situaciones de conflicto, Tirant Lo Blanch, Valencia, 2013, pp. 132 y ss. 
produce en el ámbito de la asistencia letrada. Tanto por las exigencias de las distintas leyes procesales vigentes en los diversos órdenes jurisdiccionales como por la realidad práctica, lo más común en un procedimiento donde se ejercita el derecho fundamental de defensa es designar a un letrado para la representación del litigante.

Es por ello que la jurisprudencia constitucional se ha centrado inicialmente en examinar los límites de la libertad de expresión cuando se ejercen en el marco del derecho de defensa ejercido por los letrados en representación de sus clientes. $Y$ ha concluido, como no podía ser de otro modo, que dicha libertad se encuentra singularmente reforzada por el contexto en que se ejerce.

Así, el TC ha considerado encuadrables en el derecho fundamental a la libertad de expresión ejercido en conexión con el derecho de defensa afirmaciones tales como que en una sentencia existen "falsedades y barbaridades" (STC 65/2004, de 19 de abril) 0 que ésta es "arbitraria, infundada, caprichosa, manifiestamente ilegal, y groseramente contraria a derecho" (STC 235/2002, de 9 de diciembre).

Del mismo modo, el TC ha considerado legítimo ejercicio de los anteriores derechos fundamentales la calificación de una resolución judicial como "de todo punto arbitraria e inmotivada" y "de todo punto ajena a los más elementales principios de la normativa adjetiva y sustantiva española vigente", así como "incomprensible" y "parcial" (STC 155/2006, de 22 de mayo), si bien en el citado caso se interpretó la expresión "parcial" (posiblemente la más grave de las empleadas) no como sinónimo de prevaricación o ánimo malicioso de beneficiar a una de las partes, sino como queja por la radical falta de sustento jurídico de la resolución judicial.

Los razonamientos que llevan al TC a considerar las anteriores expresiones como constitucionalmente protegidas y a consagrar el singular ámbito de libertad de expresión de los letrados cuando se ejerce en el marco del derecho de defensa, pueden encontrarse de un modo especialmente claro y conciso en la STC 117/2003, de 16 de junio, que reza lo siguiente: En nuestra jurisprudencia se parte de que el ejercicio de la libertad de expresión en el seno del proceso judicial por los Letrados de las partes, en el desempeño de sus funciones de asistencia técnica, posee una singular cualificación, al estar ligado estrechamente a la efectividad de los derechos de defensa del art. 24 CE (STC 113/2000, de 5 de mayo, FJ 4). Consiste en una libertad de expresión reforzada cuya específica relevancia constitucional deviene de su inmediata conexión con la efectividad de otro derecho fundamental, el derecho a la defensa de la parte (art. 24.2 CE), y al adecuado funcionamiento de los órganos jurisdiccionales en el cumplimiento del propio y fundamental papel que la Constitución les atribuye (art. 117 CE). Por tales razones se trata de una manifestación especialmente inmune a las restricciones que en otro contexto habrían de operar (STC 205/1994, de 11 de julio, FJ 5). 
Consiguientemente, la finalidad del plus de libertad de expresión concedido a los letrados en este ámbito tiene un fin manifiesto: permitir que sus clientes puedan gozar de una adecuada defensa, sin que el temor a sanciones disciplinarias pueda coartar al abogado a la hora de manifestar con toda la contundencia precisa los errores o arbitrariedades del tribunal, esgrimiéndolos en los pertinentes recursos de reposición, apelación o casación a fin de que sean corregidos por el tribunal de instancia o por otro superior.

Ahora bien, precisamente de la íntima conexión entre el mayor margen de libertad de expresión de los letrados y el derecho de defensa, se derivan sus propios límites. Las expresiones del letrado que contengan una mayor contundencia y beligerancia, estarán justificadas en la medida que se dirijan a denunciar infracciones procesales o arbitrariedades provocadas por el juzgador. Pero jamás quedará amparado el insulto desnudo o los descalificativos personales que no guarden relación con los actos de irrazonabilidad 0 arbitrariedad judicial que se denuncia. En tal sentido, establece el Tribunal Constitucional en la misma STC 117/2003, de 16 de junio, que: Asimismo hemos puntualizado que la especial cualidad de la libertad de expresión del Abogado en el ejercicio de defensa de su patrocinado debe valorarse en el marco en el que se ejerce $y$ atendiendo a su funcionalidad para el logro de las finalidades que justifican su privilegiado régimen, sin que ampare el desconocimiento del respeto debido a las demás partes presentes en el procedimiento y a la autoridad e imparcialidad del Poder Judicial, que el art. $10.2 \mathrm{del}$ Convenio Europeo de Derechos Humanos erige en límite explícito a la libertad de expresión (SSTC 205/1994, de 11 de julio, FJ 5; 157/1996, de 15 de octubre, FJ 5; 226/2001, de 26 de noviembre, FJ 2; 79/2002, FJ 6; STEDH de 22 de febrero de 1989, caso Barfod).

No podemos dejar de resaltar la vinculación del anterior criterio con la doctrina del efecto desaliento consagrada por el TC en sentencias como la STC 110/2000, de 5 de mayo. En virtud de la anterior doctrina, cuando existiesen dudas sobre si el ejercicio de conductas encuadrables prima facie en un derecho fundamental ha podido llegar a superar los límites de aquél y entrar en lo sancionable, deberá realizarse la interpretación más favorable a su ubicación dentro de los límites de tal derecho.

$\mathrm{Si}$, por el contrario, no cupiese duda sobre el exceso que han supuesto tales conductas, deberán castigarse, pero sin caer jamás en el exceso o la desproporción sancionadora, pues ello provocaría un temor a la hora de ejercer los derechos fundamentales que disuadiría a los ciudadanos de hacerlo por la posibilidad de ser duramente castigados al traspasar unas fronteras (las de los límites del derecho) que en numerosas ocasiones no están claras $^{3}$.

3. T. De Domingo Pérez, "La argumentación jurídica en el ámbito de los derechos fundamentales: en torno al denominado "chilling effect" o "efecto desaliento"', Revista de estudios políticos, No 122, 2003, p. 153. 
En tal sentido, resulta evidente el efecto nocivo y demoledor para los derechos de los justiciables que implicaría el miedo de sus letrados a ejercer su defensa con toda la determinación y contundencia que el valor de los derechos e intereses en juego exige, resultando por ello esencial evitar que esa situación de temor pueda llegar a producirse 4 .

\section{3.- LIBERTAD DE EXPRESIÓN Y DERECHO DE DEFENSA EJERCIDOS SIN ASISTENCIA LETRADA EN EL ÁMBITO DEL PROCEDIMIENTO ADMINISTRATIVO SANCIONADOR: UN ANÁLISIS A LA LUZ DE LA STC 187/2015, DE 21 DE SEPTIEMBRE}

Hemos examinado la dimensión de la libertad de expresión reconocida a los letrados cuando ejercen la defensa de sus clientes. Pero ¿Puede extrapolarse dicha libertad al ciudadano cuando éste ejerce su defensa en primera persona y sin acudir a un profesional del Derecho? El TC se ha pronunciado en fechas recientas sobre una de las principales manifestaciones de esa situación: la del funcionario público que, en el marco de un procedimiento disciplinario contra su persona, asume su propia defensa.

Así, la STC 187/2015, de 21 de septiembre resuelve el recurso de amparo interpuesto por un funcionario interino que fue sancionado con suspensión de empleo y sueldo durante 23 meses por haber remitido sendos escritos a la Inspección Educativa de la Consejería de Educación de la Región de Murcia alegando que se sentía acosado por diversos funcionarios dependientes de la misma, todo ello en el marco de un expediente disciplinario previo que se había incoado a dicho funcionario por razones distintas.

El funcionario recurrente en amparo presentó ante la Inspección Educativa un escrito de fecha 3 de octubre de 2008 en el que, defendiéndose de las acusaciones objeto del primer expediente disciplinario, afirmaba ser objeto de acoso laboral y exponía una serie de hechos que consideraba prueba de ello, y que estaban directamente relacionados con la arbitraria (a su juicio) instrucción de ese primer expediente. Dicho escrito se presentó dentro del trámite de audiencia del citado expediente.

Tras ello, recibió la visita de varios inspectores de personal, por lo que remitió a la Inspección Educativa un nuevo escrito de 20 de octubre de 2008 donde se reiteraba en su afirmación de estar sufriendo acoso y razonaba que, a su entender, la visita de los inspectores formaba parte del mismo y tenía por objeto dar la falsa imagen de que no había sufrido acoso alguno, ya que los inspectores no realizaron una investigación objetiva e imparcial, sino que se limitaron a legitimar acríticamente la conducta de quienes, a su

4. J.C. Gavara de Cara, El control judicial de los medios de comunicación, Editor J.M. Bosch, Barcelona, 2015, pp. 125 y ss. 
juicio, le estaban acosando. De este modo, razonaba el funcionario, se pretendía preparar el terreno para su sanción en el marco del primer expediente, así como para la apertura de un segundo expediente disciplinario por sus manifestaciones de queja y denuncia.

En ambos escritos, el funcionario afirmaba, refiriéndose a uno de los inspectores de personal, que tenía "sed de venganza" hacia su persona, y sostenía que había cometido "chapuzas" en el tratamiento de su caso al haber enviado las notificaciones de su expediente disciplinario a otro funcionario para que él no pudiese recibirlas.

También manifestaba que una determinada autoridad estaba usando al inspector como una "marioneta" y le dirigía a través de su teléfono móvil, y se preguntaba si el inspector habría tenido algo que ver en su exclusión de unas oposiciones a las que se presentó. Del mismo modo, denunciaba que su expediente disciplinario formaba parte del acoso laboral que padecía, y que estaba seguro de que se le abriría un segundo expediente por sus denuncias (como así fue).

Finalmente, cuestionaba que la firma en un documento obrante en su expediente disciplinario perteneciese al Director de su centro o a "alguien con su firma muy parecida a la de éste", y se preguntaba, refiriéndose a una carta que se empleó para abrirle el segundo expediente disciplinario, si el inspector "se la guardó como un as en la manga para sacarla de forma inopinada, cobarde y a traición para abrirme el expediente".

Por todas estas manifestaciones, el recurrente en amparo sufrió la apertura de un segundo expediente disciplinario, cuyo resultado fue la sanción por la comisión de ocho faltas disciplinarias graves, tipificadas en los apartados e) y ñ) del art. 7.1 del Real Decreto 33/1986, de 10 de enero, que aprueba el Reglamento de régimen disciplinario de los funcionarios de la Administración del Estado. Las manifestaciones incluidas en los escritos de 3 y 20 de octubre de 2008 se consideraron constitutivas de las conductas tipificadas como grave desconsideración con otros funcionarios, y como grave atentado a la dignidad de otros funcionarios.

Disconforme con la sanción, el recurrente en amparo acudió a la jurisdicción contencioso administrativa, obteniendo en primera instancia sentencia estimatoria de sus pretensiones que anuló las sanciones impuestas. El juzgador de instancia consideró que las manifestaciones vertidas encajaban plenamente en el contenido reforzado que el derecho fundamental a la libertad de expresión posee cuando se ejerce en conexión con el derecho de defensa, pues el recurrente se estaba defendiendo en el marco de un primer expediente disciplinario con el fin de que las actuaciones arbitrarias (a su juicio) del instructor y otros funcionarios cesasen, y de que no se le sancionase injustamente.

La Administración recurrió en apelación la sentencia de instancia, y su recurso fue estimado por la STSJ de Murcia 928/2012, Sala de lo Contencioso Administrativo, de 11 de diciembre, que la revocó y mantuvo en su integridad las sanciones impuestas por la 
Administración. La Sala desautorizó el criterio del juez de instancia con el siguiente razonamiento: Para ello utiliza criterios jurisprudenciales que, como él mismo reconoce, se refieren a "los Abogados en el ejercicio de su profesión". Y para traer esos criterios a colación en este caso, parte el juez de instancia de que el apelado tenía "la íntima convicción de que está siendo víctima de acoso laboral..." (sic.). Y, claro, por eso estaba defendiéndose. Pero este argumento no es sostenible, ya que debe entenderse que la existencia de una conducta tan indeseable como el acoso laboral, no puede sustentarse sino en hechos y nunca en una "íntima convicción". No estaba pues defendiéndose de nada y contra tantas personas, cuando el apelado vertió insultos y descalificaciones, que merecen el reproche proporcionado de que fueron objeto.

Como se observa, la sentencia era doblemente errónea. En primer lugar, parecía establecer que la jurisprudencia aplicable a los letrados para ampliar el marco de su libertad de expresión cuando ejercen la defensa de sus clientes, no es extrapolable al ciudadano que asume en primera persona su defensa. Tal tesis fue desautorizada por el TC, tal y como expondremos más adelante.

En segundo lugar, la sentencia viene a decir que si se pretende ejercer el derecho de defensa en relación con un posible acoso, y éste no consigue probarse, en realidad no se está ejerciendo tal derecho porque el actuante "no estaba pues defendiéndose de nada". Tal argumentación resulta singularmente difícil de asumir, pues implicaría que los letrados nunca podrían ampararse en el ejercicio del derecho de defensa cuando perdiesen un caso, pues no habrían logrado probar la veracidad y corrección de su postura.

Tras interponer el pertinente incidente de nulidad de actuaciones, el recurrente en amparo presentó el recurso ante el TC, que lo admitió a trámite dando lugar a la sentencia que nos ocupa. Como dato anecdótico debemos reseñar que el motivo de la admisión a trámite fue la originalidad del caso y la falta de pronunciamientos del TC sobre supuestos como el presente, encontrándose ahí su especial trascendencia constitucional. Así, dice la sentencia que: $(P)$ ueden calificarse de aislados y no muy recientes los pronunciamientos que atañen a los límites del derecho de libertad de expresión cuando con él se trata no ya de defender un derecho o interés legítimo, sino de ejercer el derecho de defensa frente al ius puniendi de la Administración y, más en concreto, cuando se trata de personas unidas a ella por una relación de sujeción especial (SSTC 120/1996, de 8 de julio, y 102/2001, de 23 de abril).

Nuestro pronunciamiento, sin duda, debe enmarcarse en esta última categoría, ya que lo que reclama el demandante de amparo es la aplicación del canon constitucional de la libertad de expresión que asiste a los abogados, a quienes ejercen este derecho cuando se encuentran inmersos en un procedimiento disciplinario, en el que el titular de la libertad de expresión actúa sin asistencia de Letrado. Precisamente por residir en ello la especial trascendencia 
constitucional de este recurso de amparo, abordaremos en primer lugar la lesión del art. 20 CE.

No podemos dejar de lamentar que el amparo a un ciudadano por parte del TC a causa de la vulneración de un derecho fundamental no dependa de la efectiva violación del derecho, sino de factores ajenos a la misma como la originalidad del caso. Resulta inconcebible que el máximo garante de la Constitución pueda permanecer inactivo ante la vulneración de preceptos tan singularmente esenciales de la misma como son los que regulan los derechos fundamentales, y cuya plena protección se le ha encomendado expresamente en el art. 53.2 CE.

Pero, por desgracia, esto sucede en los innumerables casos en los que, habiéndose interpuesto un recurso de amparo cuyo relato de hechos aporta elevadísimos indicios de vulneración de un derecho fundamental, se inadmite a trámite porque (por ejemplo) no es un caso original o no afecta a un elevado número de ciudadanos en la misma situación.

Por todo lo anterior, entendemos que la plena protección de los derechos fundamentales de los ciudadanos por el máximo órgano encargado de ello en virtud de imperativo constitucional, exige la modificación del art. 50.1 LOTC para eliminar el requisito de la especial trascendencia constitucional ${ }^{5}$ como premisa para la admisión a trámite de los recursos de amparo.

Volviendo al examen de la sentencia, el TC comienza corrigiendo el grave error de la Sala de lo Contencioso Administrativo del TSJ de Murcia según el cual si no se termina acreditando la veracidad de una manifestación realizada en el marco del ejercicio del derecho de defensa, ésta no quedará amparada por el contenido del mismo. Indica la sentencia que: Dado que la Sentencia de la Sala viene a afirmar, para rechazar el argumento en que se había basado la Sentencia de instancia, que «debe entenderse que la existencia de una conducta tan indeseable como el acoso laboral, no puede sustentarse sino en hechos, nunca en una íntima convicción», no estará de más recordar ahora la doctrina constitucional acuñada sobre la distinción entre la libertad de expresión [art. 20.1 a) CE] y la libertad de información [art. 20.1 d) CE]. En resumen, la libertad de expresión «tiene su campo de proyección más propio en la manifestación de valoraciones o juicios que, es evidente, quedan al margen de toda confirmación o desmentido fáctico», mientras que la

5. Son numerosos los autores que comparten las críticas sobre la actual configuración del recurso de amparo constitucional. A modo de ejemplo, Andrés De La Oliva Santos reprocha que con la actual LOTC, "las violaciones de libertades y derechos fundamentales referidos en el art. 53.2 CE dejan de ser relevantes para el TC, dejan de ser amparables por el TC, si no son constitucionalmente interesantes" (A. De La Oliva Santos, "La perversión jurídica del amparo constitucional en España", en Justicia y derecho tributario: homenaje al profesor Julio Banacloche Pérez, La Ley, Madrid, 2008, p. 407). 
libertad de información «se orienta, sobre todo, a la transmisión o comunicación de lo que se tienen por hechos -susceptibles, entonces, de contraste, prueba o mentís-» (STC 63/2015, de 13 de abril, FJ 2).

Es decir, la defensa en el marco de un procedimiento judicial o administrativo implica la construcción de un discurso argumentativo donde se imbrican valoraciones jurídicas y fácticas, así como también un relato de hechos. Pero el elemento clave y preponderante se encuentra en la manifestación de opiniones, valoraciones y juicios, por lo que queda protegido por la libertad de expresión y, como es obvio, no resulta preciso acreditar la veracidad de los hechos esgrimidos en demanda para entender aplicable este derecho.

Continúa exponiendo la sentencia el especial contexto en que se ejerce la libertad de expresión en un supuesto como el presente, donde no se busca difundir ideas ante la ciudadanía, sino exponerlas en un círculo sumamente reducido (en nuestro caso la Inspección Educativa) a los efectos de ejercer la defensa para evitar una sanción. Así, indica que: Se trata, por tanto, de manifestaciones que no responden a una finalidad informativa general o destinada a un círculo más o menos amplio de destinatarios (STC 241/1999, de 20 de diciembre, FJ 3), sino dirigidas a hacer valer un derecho subjetivo del demandante cual es la defensa frente al ius puniendi de la Administración. En otras palabras, el art. 20.1 a) CE abarca el juicio crítico acompañado por una inclinación o vocación restringida al terreno de lo no difundido como es propio de este derecho cuando se conecta con la efectividad de otros derechos fundamentales y en concreto con el derecho a la defensa del art. 24.2 CE (STC 241/1999, FJ 3).

Destaca a continuación el TC que ciertamente la libertad de expresión de algunos funcionarios puede verse restringida en relación con la libertad de expresión de un ciudadano común. Así sucede, por ejemplo, con los integrantes de las Fuerzas y Cuerpos de Seguridad del Estado ${ }^{6}$. Pero tales restricciones nunca afectarán al funcionario cuando ejerza dicha libertad en conexión con su derecho de defensa. En tal sentido, dispone la sentencia que: En lo que respecta al derecho de defensa en el procedimiento disciplinario, este Tribunal no ha establecido modulación o matización alguna debida a la relación de la especial sujeción en la que se encuentra el funcionario, pues el entendimiento de la libertad especialmente reforzada por su inmediata conexión con otro derecho fundamental, es perfectamente trasladable a los supuestos en los que es el propio afectado quien asume su defensa, por no ser preceptiva la asistencia letrada.

De este modo, el TC establece que el derecho de defensa del funcionario no se ve minorado por su situación de especial sujeción y, seguidamente, establece el canon aplicable a los supuestos en que el

6. A. Magdaleno Alegría, Los límites de las libertades de expresión e información en el Estado social y democrático de Derecho. Congreso de los Diputados, Madrid, 2006, pp. 418 y ss. 
funcionario ejerce conectadamente $y$ en primera persona sus derechos fundamentales a la libertad de expresión y a la defensa en el marco de un procedimiento sancionador: En definitiva, cuando la libertad de expresión sirve al derecho fundamental consagrado en el art. 24.2 CE, el canon aplicable debe ser el que este Tribunal tiene acuñado en relación al contenido de la libertad en el ejercicio de la defensa letrada, que la hace especialmente inmune a sus restricciones para la preservación de otros derechos $y$ bienes constitucionales, estando condicionado, claro está, a esa funcionalidad de defensa y a que no suponga un detrimento desproporcionado de los derechos de los demás partícipes en el proceso y la integridad del proceso mismo (STC 39/2009, FJ 3).

(...) Es por ello que la interpretación de los preceptos que tipifican infracciones disciplinarias que generan un riesgo para la libertad de expresión debe realizarse en el sentido de considerar que son sólo sancionables las manifestaciones o actitudes insultantes o descalificadoras, cuando esta se encuentra reforzada por ejercerse en el contexto del procedimiento disciplinario y con el ánimo de defensa, que es lo que este Tribunal tiene declarado para las sanciones impuestas a Letrados por las expresiones vertidas en el ejercicio del derecho de defensa (STC 231/2012, de 10 de diciembre, FJ 4).

Así, en el presente caso existía un nexo innegable entre las expresiones del funcionario y su voluntad de defenderse en el marco de un expediente disciplinario que, a su entender, estaba siendo arbitrariamente tramitado en su perjuicio, buscando prevenir la injusta imposición de sanciones derivadas del mismo y, además, la apertura de un segundo expediente disciplinario que, como se temía, terminó iniciándose.

Por tal causa, y puesto que las expresiones no incluían insultos o descalificaciones gratuitas de ningún tipo, estando todas ellas orientadas a la denuncia de un mal proceder administrativo que el funcionario percibía como cierto, y que iba a derivar en la imposición de sanciones a su persona, el TC considera que todo lo manifestado por el funcionario en sus dos escritos queda protegido por el derecho fundamental a la libertad de expresión puesto en conexión con el derecho de defensa.

Finalmente, concluye el TC su razonamiento destacando que, en estos supuestos, resultará igualmente aplicable la doctrina del efecto desaliento ya comentada, y ello con las siguientes palabras: $A$ ello debemos aún añadir que, como este Tribunal afirmó en las SSTC 110/200, de 5 de mayo, FJ 5, y 104/2011, de 20 de junio, FJ 6, la dimensión objetiva de los derechos fundamentales, su carácter de elementos esenciales del Ordenamiento jurídico permite afirmar que no basta con la constatación de que la conducta sancionada sobrepasa las fronteras de la expresión constitucionalmente protegida, sino que ha de garantizarse que la reacción frente a dicha extralimitación no pueda producir «por su severidad, un sacrificio innecesario o desproporcionado de la libertad de la que privan, o un 
efecto disuasor o desalentador del ejercicio de los derechos fundamentales implicados en la conducta sancionada».

Y es que la interpretación extensiva o irrazonablemente dura de los tipos sancionadores en casos como el que nos ocupa, generalizaría el miedo en un contexto donde el titular del derecho de defensa (funcionario que se enfrenta a una posible sanción con las terribles consecuencias que ello implica para su futuro) sufre una singular vulnerabilidad, lo cual derivaría en un temor a ejercer plenamente el derecho de defensa que dañaría gravemente la vigencia práctica del derecho fundamental.

Por todo lo manifestado, el TC concede su amparo al recurrente y deja sin efecto las sanciones impuestas.

\section{4.- A MODO DE CONCLUSIÓN: FACTIBILIDAD DE UNA POSIBLE EXTRAPOLACIÓN DEL CRITERIO JURISPRUDENCIAL CONSAGRADO EN LA STC 187/2015, DE 21 DE SEPTIEMBRE AL RESTO DE PROCEDIMIENTOS ADMINISTRATIVOS Y JUDICIALES DONDE EL CIUDADANO PUEDE EJERCER SU DEFENSA EN PRIMERA PERSONA}

A nuestro entender, la línea jurisprudencial que el TC acuña con la anterior sentencia, no puede ceñirse al supuesto en que el funcionario se defiende frente al ius puniendi de la Administración en el marco de un expediente disciplinario. No hay razón alguna para excluir los numerosos supuestos donde el justiciable o administrado puede ejercer su defensa en primera persona debido a que la correspondiente norma procesal no impone la asistencia letrada obligatoria.

Tal es el caso (entre otros) del trabajador que decide defenderse a sí mismo en un procedimiento judicial por despido, ya que la Ley 36/2011, de 10 de octubre, reguladora de la Jurisdicción Social, le permite hacerlo sin acudir a un letrado. Y lo mismo sucede con el administrado que presenta sus alegaciones en el marco de un procedimiento administrativo sancionador derivado de una infracción de tráfico con su vehículo.

Y es que resultaría notoriamente irrazonable y discriminatorio no extrapolar al ciudadano en toda su dimensión el plus de libertad de expresión del que gozan los letrados para la defensa de sus clientes. Esa libertad adicional que se concede al letrado tiene como causa principal la correcta defensa de los intereses de sus clientes. Por tanto, sería absurdo que no se concediese a los directos titulares de esos intereses cuando, en los casos donde la ley lo permite, pretendan defenderlos en primera persona y sin intermediación de un letrado.

Es más, entendemos que, a la hora de examinar los posibles excesos en que pueda incurrir un ciudadano al ejercer su derecho de defensa, debe valorarse su condición de lego en Derecho. Y ello no puede sino llevar a ampliar aun más su margen de libertad de 
expresión en relación con el de los letrados, pues es consecuencia lógica de su desconocimiento del Derecho el que pueda incurrir en expresiones que, por no saber su exacto significado, diga sin ser consciente de lo que pudieran representar.

Así, está claro que jamás podría ampararse en la libertad de expresión el insulto desnudo del justiciable hacia el juzgador, pero si (por ejemplo) un trabajador interpusiese un recurso contra un Auto de denegación de prueba anticipada en el marco de un procedimiento seguido ante la jurisdicción social, y en dicho escrito afirmase que "el juez está actuando de un modo injusto al denegar la prueba, y lo sabe", entendemos que nunca podría acusársele de imputar un delito de prevaricación al juzgador, pues su desconocimiento del Derecho le lleva a usar esas palabras para mostrar su firme oposición a una resolución que considera injusta y que no entiende cómo puede haberse dictado.

En el anterior ejemplo, el ciudadano expresa que, siendo a su juicio tan claramente injusta la resolución judicial, le resulta incomprensible que el juez no se dé cuenta de ello, pero sin que necesariamente esté afirmando que el juez ha dictado su resolución maliciosamente, y sin saber la gravedad que podrían tener sus palabras si se interpretasen en el sentido de que está acusando al juez de dictar una resolución injusta a sabiendas y dolosamente.

Por todo lo anterior, y sintetizando lo expuesto, entendemos que el criterio exteriorizado por el TC en la sentencia que nos ocupa excede el concreto supuesto al que se refiere, y debe considerarse aplicable a todos los supuestos en que el ciudadano ejerza su derecho de defensa sin la intermediación de letrado. 